\title{
LEUCEMIA E GASTOS HOSPITALARES: UMA ANÁLISE DO IMPACTO ECONÔMICO PARA O SISTEMA PÚBLICO DE SAÚDE DE MONTES CLAROS, MG
}

\author{
LEUKEMIA AND HOSPITAL EXPENSES: AN ANALYSIS OF THE ECONOMIC IMPACT IN \\ MONTES CLARO, $M G$ \\ João Víctor Cordeiro Rodrigues ${ }^{\mathrm{a}^{*}}$, Adelhane Martins Sanches ${ }^{\mathrm{b}^{*}}$, \\ Amanda Teixeira Duque de Oliveira ${ }^{\mathrm{c}^{*}}$, Leander de Almeida Ribeiro ${ }^{\mathrm{d}^{*}}$, Rosa Maria Ruas Paraíso ${ }^{\mathrm{e}^{*}}$, \\ Dorothéa Schimidt França ${ }^{\mathrm{f}^{*}}$
}

\author{
joaovictor1711@hotmail.coma , dehmartt@hotmail.com ${ }^{\mathrm{b}}$, amandaatdo@outlook.comc, legas017@gmail.com ${ }^{\mathrm{d}}$, rmrparaiso@yahoo.com.bre, \\ dorotheafranca@gmail.com ${ }^{\mathrm{f}}$ \\ Faculdades Integradas Pitágoras de Montes Claros - FIPMoc
}

Data de recebimento do artigo: $27 / 11 / 2018$

Data de aceite do artigo: 18/02/2019

\section{RESUMO}

Introdução: A leucemia, proliferação neoplásica generalizada ou acúmulo de células hematopoiéticas, que há ou não envolvimento do sangue periférico, é um câncer complexo, cujo tratamento objetiva destruir as células leucêmicas visando à produção de células normais pela medula óssea, o que é dispendioso para o Sistema Único de Saúde (SUS). Objetivo: Analisar os impactos econômicos que a leucemia demanda para o SUS em Montes Claros, MG, no período de janeiro de 2010 a janeiro de 2018, considerando o sexo masculino e feminino e as diferentes faixas etárias. Metodologia: Pesquisa descritiva, sistemática, longitudinal e quantitativa. Dados coletados referentes a janeiro de 2010 a janeiro de 2018, sobre gastos públicos com procedimentos hospitalares relacionados à leucemia, na plataforma DATASUS. Amostra composta pela população do município de Montes Claros - MG acometida pela neoplasia e que geraram algum gasto hospitalar. Indivíduos de ambos os sexos acometidos pela afecção e de todas as idades foram incluídos. Não houve critérios de exclusão. Resultados: Os gastos hospitalares com leucemia em Montes Claros foram variáveis, não apresentando um padrão que acompanha o número total de internações no período, o que denota a individualidade de cada paciente. Considerando o sexo, os homens foram mais dispendiosos e, com relação à faixa etária, sem distinção de sexo, os indivíduos de 0 a 19 anos. Conclusão: A leucemia demanda muita atenção e investimento por parte do Sistema Único de Saúde, em que a busca por um tratamento eficaz que englobe a individualidade de cada paciente gera grandes impactos econômicos para o SUS.

Palavras-chaves: Leucemia; gastos em saúde; Sistema Único de Saúde

\section{ABSTRACT}

Introduction: The Leukemia, generalized neoplastic proliferation or accumulation of hematopoietic cells, which can be found or not found in peripheral blood, is a complex cancer, its treatment goal is to destroy the cells after a growth with the production of normal cells by the boné marrow, which is expensive for the Unified Health System (SUS). Objective: To analyze the effects of the leukemia impacton SUS in Montes Claros, MG, from January 2010 to January 2018, considering the male and female gender and the different age groups. Methodology: Descriptive, systematic, longitudinal and quantitative research. Data collected from January 2010 to January 2018, on hospital expansion with hospital data related to leukemia, on the DATASUS platform. Sample is composed by the population of the municipality of Montes Claros - MG, affected by the neoplasm and managed some hospital event. Individuals of both sexes affected by the condition and all the ages were included. There was no exclusion criterion. Results: Hospital expenseswithleukemia in Montes Claros werevariable, notpresenting a patternthataccompaniesthe total hospitalizations in theperiod, which indicates the individuality of each patient. Considering sex, men were more expensive and, with respect to age, without the difference of sex, the individual from 0 to 19 years. Conclusion: A leukemia of great investment and investment by the Unified Health System, in which a search for na effective effort that encompasses a high cost individuality for the SUS.

Keywords: Leukemia; health expenditures; Unified Health System 


\section{Introdução}

A leucemia pode ser definida como uma proliferação neoplásica generalizada ou acúmulo de células hematopoiéticas em que pode ou não ocorrer envolvimento do sangue periférico ${ }^{1}$. A patologia acomete qualquer classe de leucócitos das linhagens granulocítica, monocítica ou mieloide, sendo que podem sofrer transformação maligna e, dependendo do seu curso clínico, pode ser aguda ou crônica ${ }^{2}$.

Leucemias agudas afetam linhagens celulares imaturas ou células blásticas e, quando não diagnosticadas e tratadas, resultam em rápida debilidade no estado geral do indivíduo ${ }^{2}$. Geralmente, as células leucêmicas extravasam para o sangue, podendo ser vistas em grande número e, na fase crônica da doença, essas células também podem invadir o fígado, baço, linfonodos e outros tecidos ${ }^{3}$.

Em relação à forma de diagnóstico da doença, conforme dados do INCA ${ }^{4}$, é fundamental a utilização do mielograma e da punção lombar. O mielograma é feito através da avaliação da medula óssea e indica se, morfologicamente, as células leucêmicas foram erradicadas da medula, provocando um estado de remissão do quadro clínico. Já na punção lombar realiza-se o exame citológico da aspiração, e é utilizado também para injeções de quimioterapia, servindo de profilaxia para as células leucêmicas no Sistema Nervoso Central (SNC), ou para destruí-las caso já existam.

O tratamento do câncer tem o intuito de destruir as células leucêmicas para que então, a medula óssea passe a produzir células normais, por meio da associação entre medicamentos, controle das complicações e prevenção de doenças do sistema nervoso central (SNC) ${ }^{4}$. Entre as modalidades de tratamento a quimioterapia é a mais frequente, podendo ser associada à radioterapia, cirurgia, imunoterapia e hormonioterapia. O protocolo de tratamento é instituído de acordo com o tipo de tumor, seu comportamento biológico, localização, extensão da doença, idade e condições gerais do paciente ${ }^{5}$.

Nesse sentido, o câncer possui alta complexidade, o que ressalta a necessidade da implementação de medidas de prevenção e proteção, diagnóstico precoce, oferecendo um tratamento adequado e abrangente, com o menor risco de sequelas, criando condições dignas para pacientes em fase terminal, bem como oferecer meios de reabilitação física, psíquica e social, o que pode tornar mais dispendiosas as despesas hospitalares. Dessa forma, este trabalho objetiva Analisar os impactos econômicos que a leucemia demanda para o Sistema Único de Saúde (SUS) em Montes Claros, MG, no período de janeiro de 2010 a janeiro de 2018, considerando o sexo masculino e feminino e as diferentes faixas etárias.

\section{Metodologia}

Trata-se de uma pesquisa descritiva, sistemática, com delineamento longitudinal na coleta de dados e abordagem quantitativa destes acerca dos gastos hospitalares com Leucemia no município de Montes Claros-MG. A pesquisa foi iniciada com uma revisão narrativa com enfoque nos aspectos gerais, epidemiologia, fisiopatologia, diagnóstico e tratamento, para dar embasamento teórico a pesquisa.

Em seguida definiu-se a amostra, que foi composta pelos indivíduos acometidos pela Leucemia, que fizeram tratamento ou gerou algum gasto decorrente dessa enfermidade nos hospitais de Montes Claros. Assim, tornou-se possível estimar a realidade municipal quanto aos gastos hospitalares no que tange esse tema. Nessa amostragem, os critérios de inclusão foram todos os indivíduos do sexo feminino e masculino que gerou algum gasto hospitalar em Montes Claros acometidos pela Leucemia, de todas as idades, residentes ou não nesse município, registrados na plataforma utilizada como base do trabalho. Não houve critérios de exclusão.

A pesquisa foi realizada com base na plataforma do DATASUS, que é o departamento de informática do Sistema Único de Saúde (SUS), cuja incumbência é coletar, processar e divulgar informações sobre a saúde no Brasil. Coletaram-se dados que abarcam os anos de 2010 á janeiro de 2018, sobre gastos hospitalares com Leucemia, estratificação dos gastos de acordo com o sexo e a faixa etária (0-19 anos, 20-49 anos, 50-79 anos, 80 anos ou mais). O período da coleta de dados ocorreu no mês de setembro de 2018.

A aprovação do Comitê de Ética foi dispensada nessa pesquisa por se tratar de dados de à caracte- 
rística aberta, impossibilitando a identificação dos sujeitos, conforme a Resolução 466/12 do Conselho Nacional de Saúde.

Os resultados encontrados foram registrados e analisados através de planilhas eletrônicas (Excel), para melhor seleção e comparação das informações coletadas, possibilitando a construção da discussão e resultados da pesquisa.

\section{Resultados e discussão}

O câncer possui alta complexidade e o seu tratamento deve ser abrangente, demandando atenção tanto para as necessidades físicas, quanto para as necessidades psicológicas e sociais, incluindo a participação da família, numa abordagem ambulatorial e hospitalar ${ }^{5,6}$. Para o tratamento, são várias as modalidades, sendo a quimioterapia a mais frequente, podendo ser associada à radioterapia, cirurgia, imunoterapia e hormonioterapia ${ }^{5}$. Dessa forma, há uma demanda de gastos para o sistema público, relacionada com os procedimentos terapêuticos realizados.

O tratamento é feito em fases, em que a primeira consiste da remissão da doença com o uso de medicamentos e, a partir de então, o tratamento varia de acordo o tipo de leucemia, podendo durar menos de um ano na mieloide, ou mais que dois anos na linfoide ${ }^{4}$. As transfusões de sangue auxiliam no tratamento da leucemia, principalmente nas fases iniciais da doença, ofertando-lhe hemácias e plaquetas enquanto a medula óssea não realizada adequada hematopoiese, de modo que são feitas quase diariamente, aumentando os gastos do tratamento ${ }^{2,4}$. Além disso, o transplante de medula óssea, procedimento caro e de alta complexidade, tanto para o paciente quanto para a equipe hospitalar, é uma alternativa a ser considerada ${ }^{4,7}$.

Tabela 1. Gastos hospitalares com leucemia em Montes Claros, MG, no período de janeiro de 2010 a janeiro de 2018.

\begin{tabular}{cc}
\hline ANO & VALOR GASTO \\
\hline $\mathbf{2 0 1 0}$ & $275.582,23$ \\
$\mathbf{2 0 1 1}$ & $226.554,86$ \\
$\mathbf{2 0 1 2}$ & $267.444,63$ \\
$\mathbf{2 0 1 3}$ & $254.984,81$ \\
$\mathbf{2 0 1 4}$ & $307.028,98$
\end{tabular}

\begin{tabular}{cc}
$\mathbf{2 0 1 5}$ & $529.981,65$ \\
$\mathbf{2 0 1 6}$ & $475.327,73$ \\
$\mathbf{2 0 1 7}$ & $471.204,46$ \\
$\mathbf{2 0 1 8}$ & $38.592,97$ \\
TOTAL NO PERÍODO & $2.846 .702,32$ \\
\hline
\end{tabular}

Com base nos dados da Tabela 1, os gastos hospitalares com leucemia em Montes Claros foi extremamente variável. $\mathrm{O}$ ano de 2015 foi o mais oneroso para o sistema público de saúde do município, evidenciando um total de $\mathrm{R} \$ 529.981,65$, seguido do ano 2016 e 2017, que apresentaram, respectivamente, um gasto de $\mathrm{R} \$ 475.327,73 \mathrm{e}$ $\mathrm{R} \$ 471.204,46$. Esses valores variam conforme a singularidade de cada paciente, tendo em vista que a abordagem terapêutica da leucemia é muito complexa, o que é evidenciado ao realizar a comparação do número de internações hospitalares do período. $\mathrm{O}$ ano que apresentou maior número de internações foi 2017 num total de 109. Todavia, o ano mais dispendioso para o município foi 2015 , que apresentou 91 internações hospitalares. A mesma diferença entre gastos e internações pode ser observada nos anos de 2010 e 2012 , sendo que 2010, com 29 internações, apresentou um gasto total de $\mathrm{R} \$ 275.582,23$, ao passo que 2012, num total de $\mathrm{R} \$ 267.444,63$, obteve 58 internações hospitalares. Destaca-se que o ano 2018 apresentou um valor anual gasto muito abaixo dos demais anos analisados em virtude da análise ter sido restrita apenas ao mês de janeiro. Todavia, quando se observa o gasto mensal médio dos demais anos do período analisado, o valor é semelhante aos anos 2016 e 2017, e ultrapassa o valor mensal médio dos anos 2011 e 2012, o que denota uma ausência de padrão dos gastos com serviços hospitalares referentes à leucemia.

A pesquisa também buscou realizar uma comparação dos gastos hospitalares com leucemia considerando os indivíduos acometidos. O protocolo de tratamento é instituído de acordo com o tipo de tumor, seu comportamento biológico, localização, extensão da doença, idade e condições gerais do paciente, não havendo, portanto, distinção para os sexos $^{5}$. De modo geral, no período analisado, os homens foram mais dispendiosos para o sistema de saúde público montes-clarense, apresentado $\mathrm{R} \$ 1.533 .323,95$, correspondente a 311 interna- 
ções, e as mulheres, num total de 262 internações, apresentaram um gasto de $\mathrm{R} \$ 1.313 .378,37$. $\mathrm{O}$ ano mais oneroso para os pacientes do sexo masculino foi 2015 , evidenciando um total de $\mathrm{R} \$ 316.748,76$, e para as mulheres foi 2017 , com $\mathrm{R} \$ 283.244,68$.

Gráfico 1. Gastos hospitalares, por sexo, no período de janeiro de 2010 a janeiro de 2018.

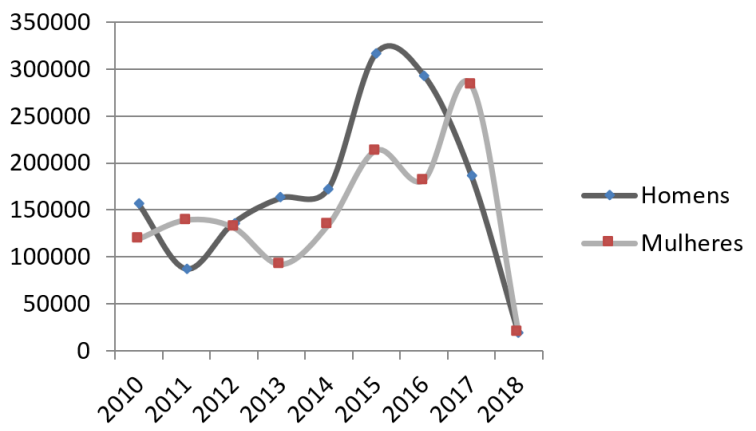

Conforme pode ser evidenciado no gráfico 1, os homens sempre apresentaram maiores gastos que as mulheres, com exceção dos anos de 2011, 2017 e o mês de janeiro de 2018. Ressalta-se que, assim como os gastos gerais apresentados, homens e mulheres também não apresentam um padrão de gastos diretamente proporcionais ao número de internações, o que evidencia que a abordagem terapêutica varia conforme a singularidade de cada paciente.

Ao comparar os gastos de serviços hospitalares com leucemia em Montes Claros, considerando a faixa etária de 0-19 anos; 20-49 anos; 50-79 anos e 80 anos ou mais no período de janeiro de 2010 a janeiro de 2018, nota-se que o maior gasto é destinado a primeira faixa etária citada, totalizando nesse período um dispêndio de $\mathrm{R} \$ 1529701,35$, e o menor gasto fica a cargo da faixa etária de 80 anos ou mais, com gasto de $\mathrm{R} \$ 48260,47$.

Esse maior gasto entre crianças e adolescentes (0-19 anos) se deve a leucemia ser o tipo de neoplasia mais comum entre essa população no mundo, representando cerca de $20 \%$ de todos os tumores que ocorrem abaixo dos 20 anos. As leucemias correspondem a $26 \%$ em crianças e adolescentes de 0 a 19 anos, no Brasil ${ }^{7,8}$. Destaca-se, ainda, que a leucemia mieloide aguda (LLA) é o tipo mais comum de tumor infantil, constituindo cerca de um terço de todas as neoplasias malignas da criança9.

Ainda, o que também corrobora para justificar o maior gasto na faixa etária pediátrica é devido às complicações do paciente leucêmico, que decorre da imunossupressão originada da própria doença e/ou induzida pela quimioterapia, uso de corticoesteroides, uso de cateteres ou antibióticos de amplo espectro e, além disso, pacientes pediátricos ainda apresentam alterações de caráter fisiológico na imunidade de forma que, com o sistema imune deficitário, há o aumento da suscetibilidade a infecções, as quais são importantes causas de morbidade e mortalidade ${ }^{10}$, gerando maiores gastos hospitalares relacionados à leucemia nessa faixa etária.

Tabela 2. Comparação dos gastos de serviços hospitalares com leucemia em Montes Claros, MG, considerando a faixa etária - 0-19 anos; 20-49 anos; 50-79 anos e 80 anos ou mais,

no período de janeiro de 2010 a janeiro de 2018.

\begin{tabular}{|c|c|c|c|c|}
\hline \multirow[b]{2}{*}{ ANO } & \multicolumn{4}{|c|}{ VALOR GASTO } \\
\hline & 0-19 ANOS & 20-49 ANOS & 50-79 ANOS & $\begin{array}{c}80 \text { ANOS OU } \\
\text { MAIS }\end{array}$ \\
\hline 2010 & $140.580,85$ & $89.486,37$ & $28.112,05$ & $17.402,96$ \\
\hline 2011 & $105.052,09$ & $83.126,34$ & $33.943,29$ & $4.433,14$ \\
\hline 2012 & $116.412,09$ & $86.197,16$ & $64.835,38$ & - \\
\hline 2013 & $149.904,96$ & $47.932,47$ & $53.184,72$ & $3.962,66$ \\
\hline 2014 & $207.288,06$ & $60.774,79$ & $26.479,66$ & $12.486,47$ \\
\hline 2015 & $291.524,58$ & $145.916,04$ & $86.753,01$ & $5.788,02$ \\
\hline 2016 & $329.347,37$ & $99.100,68$ & $46.655,97$ & 223,71 \\
\hline 2017 & $174.942,28$ & $227.088,64$ & $65.700,78$ & $3.472,76$ \\
\hline 2018 & $14.649,07$ & $12.121,40$ & $11.331,75$ & 490,75 \\
\hline TOTAL NO PERÍODO & $1.529 .701,35$ & $851.743,89$ & $416.996,61$ & $48.260,47$ \\
\hline
\end{tabular}


De acordo com a Tabela 2 percebe-se que o maior gasto foi no ano de 2016 na faixa etária de 0-19 anos com um valor de R\$329.347,37. Em contrapartida, nesse mesmo ano, o gasto realizado com pacientes de 80 anos ou mais foi de $\mathrm{R} \$ 223,71$, ficando a abaixo do gasto de 2018, já que a pesquisa só apresenta o gasto do mês de janeiro, no valor de R\$490,47. Na faixa etária de 20-49 anos, o ano mais oneroso foi o de 2017, com gasto equivalente a $\mathrm{R} \$ 227.088,64$, já na faixa de $50-79$ anos o ano mais dispendioso foi o de 2015 com gasto de $\mathrm{R} \$ 86.753,01$. Ressalta também que a média anual de gastos com leucemia nos pacientes com 80 anos ou mais é de $\mathrm{R} \$ 6.032,55$, no entanto, o ano de 2010, alcançou um gasto de $\mathrm{R} \$ 17.402,96$, sendo o mais oneroso dentre os 8 anos pesquisados.

Ao considerar o ano de 2018, em que foi colhido apenas o gasto referente ao mês de janeiro, nota-se que o esse valor gasto com os pacientes de 0-19 anos é 29,85 vezes maior do que o gasto com pacientes de 80 anos ou mais, o que reforça ainda mais a menor taxa de incidência nessa faixa etária, em torno de 3,7/100 mil pessoas por ano ${ }^{11}$.

Ao analisar os gastos com internação para quimioterapia de leucemia aguda e crônica agudizada em Montes Claros-MG, no período de janeiro de 2010 a janeiro de 2018 , computa um valor gasto de $\mathrm{R} \$ 2.098 .482,18$. No caso específico da leucemia, o semestre inicial de tratamento é crítico, uma vez que concentra internações mais longas e terapêutica mais agressiva ${ }^{12}$, o que justifica os gastos para esse fim.

O gasto com internação para quimioterapia foi menor no ano de 2011, R $\$ 79.083,39$, e o maior foi no ano de 2015, com R $\$ 473.023,51$. A média anual desse gasto ficou em torno de $\mathrm{R} \$ 262.310,27$. E a média de gasto mensal ficou em torno de $\mathrm{R} \$ 21.859,18$, valor mais alto do que o mês de janeiro de 2018 que obteve R\$ 19.798,55 de gasto. Esses valores podem ser evidenciados na Tabela 3.

Tabela 3. Gastos com internação para quimioterapia de leucemia aguda e crônica agudizada, em Montes Claros, MG, no período de janeiro de 2010 a janeiro de 2018.

\begin{tabular}{cc}
\hline ANO & VALOR GASTO \\
\hline 2010 & $189.165,34$ \\
2011 & $79.083,39$ \\
2012 & $171.358,95$
\end{tabular}

\begin{tabular}{cc}
$\mathbf{2 0 1 3}$ & $221.407,83$ \\
$\mathbf{2 0 1 4}$ & $211.319,61$ \\
$\mathbf{2 0 1 5}$ & $473.203,51$ \\
$\mathbf{2 0 1 6}$ & $394.108,58$ \\
$\mathbf{2 0 1 7}$ & $339.036,42$ \\
$\mathbf{2 0 1 8}$ & $19.798,55$ \\
TOTAL NO PERÍODO & $2.098 .482,18$ \\
\hline
\end{tabular}

Ao relacionar a tabela 2 com a tabela 3 , observa-se que os gastos com internação para quimioterapia estão inclusos nos gastos da tabela 2. Assim, os gastos hospitalares totais com leucemia em Montes Claros são de $\mathrm{R} \$ 2.846 .702,32$, incluindo todas as faixas etárias pesquisadas, e $\mathrm{R} \$ 2.098 .482,18$ para internação para quimioterapia, restando um saldo de $\mathrm{R} \$ 748.220,14$ que foram aplicados em outras despesas e outros tratamentos referentes à leucemia, anteriormente mencionados nessa discussão.

\section{Conclusão}

A leucemia revela-se um câncer de grande complexidade e com um tratamento que demanda muita atenção e investimento por parte do Sistema Único de Saúde, seja pela da própria neoplasia quanto por complicações oriundas da imunossupressão da doença ou induzida pelo tratamento, o que é evidenciado nas diversas faixas etárias, como a pediátrica.

Um dos principais desafios consiste na realização de um tratamento eficaz que englobe a individualidade de cada paciente, visto que a abordagem com o paciente leucêmico é baseada em diversas formas, tendo como base as necessidades físicas, psicológicas e sociais, e não apenas as necessidades biológicas, gerando grandes impactos econômicos para o Sistema Único de Saúde. Conforme as pesquisas, revelam-se gastos maiores e variáveis em determinadas idades e dependente do sexo, pois o tratamento é feito de várias formas que dependerá do tipo de leucemia e de acordo as particularidades e limitações de cada paciente. Foi observado ainda, que na maior parte do tempo analisado, os indivíduos do sexo masculino acarretaram maiores gastos para o sistema público de 
saúde de Montes Claros e, quando verificado a faixa etária, percebe-se que nas faixas etárias primordiais (0-19 anos) houve maior gasto, ao passo que, os indivíduos maiores que 80 anos designam menores gastos para Sistema Único de Saúde. Desse modo, certifica-se que a busca pela qualidade de vida do paciente tem altos impactos no orçamento do Sistema Único de Saúde.

\section{REFERÊNCIAS}

1. Henry JB. Diagnósticos Clínicos e Tratamento por Métodos Laboratoriais. 2 ed. São Paulo: Manole; 1999.

2. Epstein JB; Stevenson MP. Periodontal disease and periodontal management in patients with cancer. Oral Oncology 2001;37(8):613-619.

3. Robbins SL et al. Patologia Estrutural e Funcional. 5 ed. Rio de Janeiro: Guanabara Koogan; 1996.

4. Instituto Nacional de Câncer José Alenca Gomes da Silva. Tratamento da leucemia. Montes Claros: 2018. [acesso em: 24 set. 2018]. Disponível em: http://www2.inca.gov. br/wps/wcm/connect/tiposdecancer/site/home/leucemia/tratamento

5. Costa JC, Lima RAG. Crianças/adolescentes em quimioterapia ambulatorial: implicações para a enfermagem. Revista latinoamericana de enfermagem 2002;10(3):321-333.

6. Campregher PV, Mattos VRP, Salvino MA, Santos FPS, Hamerschlak N. Tratamento bem-sucedido de leucemia mieloide aguda recorrente após transplante com duplicação interna em tandem FLT3 usando combinação de indução por quimioterapia, infusão de linfócitos de doador, soferanib e azacitidina. Relato de três casos. Einstein 2017;15(3):355-358.

7. Registro Nacional de Doadores Voluntários de Medula Óssea. Transplante de medula óssea. Montes Claros: 2018. [acesso em: 24 mar. 2018]. Disponível em: http://redome. inca.gov.br/paciente/transplante-de-medula-ossea/

8. Instituto Nacional de Câncer - INCA. Incidência. Montes Claros: 2018. [acesso em: 02 out. 2018] Disponível em: http://wwwl.inca.gov.br/wcm/incidencia/2017/pdf/incidencia.pdf.

9. Pedrosa F, Lins M. Leucemia Linfoide Aguda: uma doença curável. Revista Brasileira de Saúde Materno Infantil 2002;2(1):63-68.

10. Gabe C, Almeida DR, Siqueira LO. Avaliação de eventos infecciosos oportunistas em crianças portadoras de leucemias. Revista Brasileira Hematologia e Hemoterapia 2009;31(2):74-79.
11. Camelo RMSoares. Tratamento de Leucemia Mieloide Aguda no Idoso. Instituto Ciências Biomédicas Abel Salazar- Universidade do Porto. Porto; 2014.

12. Kohlsdorf MJ, Áderson LC. Cuidadores de crianças com leucemia: exigências do tratamento e aprendizagem de novos comportamentos. Universidade de Brasília. Estudos de Psicologia 2011;16(3):227-234.

Como citar este artigo:

Rodrigues JVC, Sanches AM, Oliveira ATD, Ribeiro LDA, Paraíso RMR. Leucemia; Gastos em Saúde; Sistema Único de Saúde. Rev. Aten. Saúde 2019;17(59):33-38. 